\title{
ROBUST AND REPEATABLE RULESET DEVELOPMENT FOR HIERARCHICAL OBJECT-BASED MONITORING OF REVEGETATION USING HIGH SPATIAL AND TEMPORAL RESOLUTION UAS DATA
}

\author{
T. G. Whiteside ${ }^{\text {a }}$ R. E. Bartolo ${ }^{\text {a }}$ \\ ${ }^{a}$ Supervising Scientist, Department of the Environment, PO Box 461, Darwin NT, 0801, (tim.whiteside, \\ renee.bartolo)@environment.gov.au
}

\begin{abstract}
KEY WORDS: UAV, Time series analysis, GEOBIA, NDVI, Ultra-high spatial resolution, Minesite rehabilitation, Monitoring
\end{abstract}

\begin{abstract}
:
The monitoring of mine site rehabilitation, particularly revegetation, involves assessing biophysical parameters over time to determine success or otherwise. Resource and logistical constraints limit the spatial and temporal scale of standard field-based monitoring methods. Unmanned aerial systems (UAS) can provide the capability to acquire optical data with coverage of an entire site at the spatial and temporal scales, suitable for the monitoring of relevant biophysical parameters. UAS data are not without challenges, such as radiometric variation between flights and sensors, different sun angles and spatial variation between dates. Therefore, a ruleset (a stepwise set of analysis algorithms) is needed that is robust and repeatable across each image regardless of spatial and radiometric variability. This study reports on the development and implementation of such a ruleset. Using a fixed wing unmanned aircraft, colour and near infrared imagery was captured over the Jabiluka mine site, located in the Northern Territory, Australia on 7 dates between April 2014 and October 2015. The imagery was radiometrically and geometrically corrected and 4 band mosaics for each date were created using photogrammetric techniques. Image analysis involved the creation of vegetation indices relevant to the data available, the segmentation of the image to delineate plants from the background and the creation of a hierarchy of objects for analysis that was consistent across all dates. Accuracy assessment showed that the GEOBIA-derived measures compared well with visual assessments of the imagery. From the analysis, the proportional cover of green plants and number of plant objects per unit area could be calculated. The time series analysis showed that proportional cover across the site varied between dates but gradually increased over time, although there was a high level of plant mortality. In addition, there was natural recruitment of volunteer plants. The same ruleset was applied to the imagery from each date demonstrating that it is robust enough to be used for further monitoring on the site. In addition, the ruleset in a modified form is currently being used for change analysis using scanned historical aerial photography.
\end{abstract}

\section{INTRODUCTION}

The monitoring of mine site rehabilitation, particularly revegetation, involves assessing biophysical parameters over time to determine success or otherwise. Resource and logistical constraints limit the spatial and temporal scale of standard fieldbased monitoring methods. One useful parameter that can be used to monitor the progress of mine site rehabilitation is the amount of vegetation foliage cover on the site. An analysis of changes in vegetation cover over time can indicate, whether a mine site is heading on a trajectory toward meeting rehabilitation goals.

Unmanned aerial systems (UAS) can provide the capability to acquire optical data with coverage of an entire site at the spatial and temporal scales, suitable for the monitoring of relevant biophysical parameters (Fletcher and Erskine 2013). UAS data are not without challenges, such as radiometric variation between flights and sensors, different sun angles and spatial variation between dates.

Geographic object-based image analysis (GEOBIA) provides a suitable framework for the analysis of ultra-high spatial resolution imagery where the above issues exist as well as for data where there exists increased within-field variability resulting from the pixel size being much smaller than the features under investigation. Time series data of a high temporal resolution are useful for monitoring the progress of rehabilitation particularly in the early stages where growth rate is rapid and tree mortality high. The project reported here describes a GEOBIA approach to monitor change in vegetation cover using a UAS data time series.

\section{METHODS}

\subsection{Study site}

Jabiluka mine site $\left(12^{\circ} 29.9^{\prime} \mathrm{S}, 132^{\circ} 54.8^{\prime} \mathrm{E}\right)$ with an area of 12 ha is located within the Jabiluka mineral lease belonging to Energy Resources of Australia (ERA) (Figure 1). The mineral lease is surrounded by the World Heritage listed Kakadu National Park approximately $300 \mathrm{~km}$ east of Darwin, Northern Territory, Australia. The local climate is monsoonal wet/dry tropics with a short intense wet season (Dec-Mar) and a longer slightly cooler dry season (April-Nov). The study site is situated on the western edge of the Arnhem Land escarpment. Although the mine was never operational, a retention pond was constructed and associated preparatory earthworks were undertaken. During 2013, the mining company conducted rehabilitation works including the removal of the retention pond and shaping of the surface. Plantings of seedlings (tubestock) occurred in two events: (1) over 3600 tubestock were planted across the site during November and December 2013; and (2) a further 4000 tubestock were planted in November 2014. 


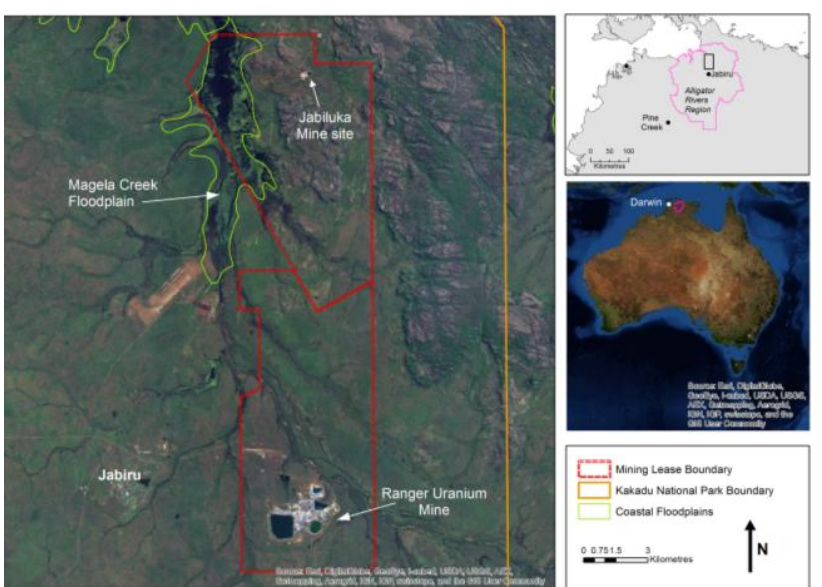

(a)

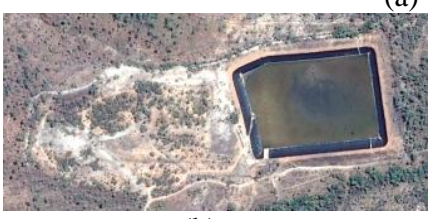

(b)

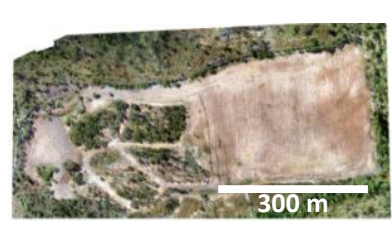

(c)
Figure 1. (a) Location of the study site. Jabiluka mine site February 2013 and after earthworks April 2014; (b) Jabiluka mine site prior to rehabilitation works (February 2013) and (c) post works (April 2014).

\subsection{Platform and sensors}

The UAS platform used for image acquisition was the Swampfox X5 fixed wing (Figure 2a) manufactured by Skycam UAV (www.kahunet.co.nz). The aircraft has a maximum all up weight of $4.7 \mathrm{~kg}$ and a payload capacity of $1.1 \mathrm{~kg}$. It is battery powered with flight duration of approximately 45 minutes maintaining a cruising airspeed of $60 \mathrm{kph}$. The sensor system (Figure 2b) used for most flights consisted of two Sony NEX5 cameras: i) red, green, blue (RGB) and ii) modified near infrared (NIR). The payload for the last flight was the Sony Nex5 RGB and a MicaSense RedEdge multispectral camera. Data was captured at a frame rate of 1 photo per 1.5 seconds along with GPS (time, heading, location and altitude) and attitude information (pitch, roll and yaw). Flights of the study site were conducted on 7 dates between 28 April 2014 and 9 October 2015. Flight patterns ensured at least $75 \%$ forward and $50 \%$ side overlap (75\% for the MicaSense flight) between images. Unfortunately, no NIR data were captured during the 23 April 2015 mission, due to sensor malfunction.

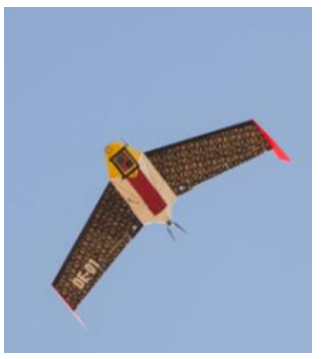

(a)

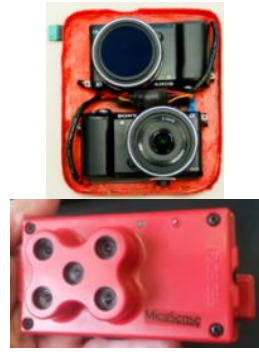

(b)
Figure 2. (a) The Skycam Swampfox aircraft and (b) the sensors used in this project: Sony NEX-5 RGB and NIR (top) and MicaSense RedEdge (bottom).
A minimum of 6 ground control points were marked on the site for each flight and their location recorded with a differential GNSS receiver (Figure 3). Also placed upon the surface were pseudo-invariant calibration panels for the radiometric correction of the imagery.

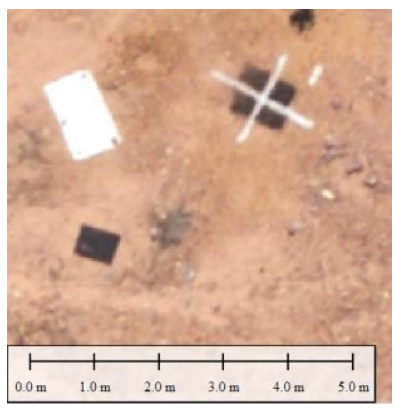

Figure 3. Ground control points and calibration panels.

\subsubsection{Data}

Table 1 shows the data captured for each flight with all fights capturing a minimum of 350 photos.

\begin{tabular}{llccc}
\hline Year & Date & RGB & NIR & RE \\
\hline 2014 & 28 April & $\checkmark$ & $\checkmark$ & \\
& 13 June & $\checkmark$ & $\checkmark$ & \\
& 23 September & $\checkmark$ & $\checkmark$ & \\
& 23 December & $\checkmark$ & $\checkmark$ & \\
\hline 2015 & 23 April & $\checkmark$ & & \\
& 10 July & $\checkmark$ & $\checkmark$ & \\
& 9 October & $\checkmark$ & $\checkmark$ & $\checkmark$
\end{tabular}

Table 1. Data captured for each date. RGB $=$ red, green, blue bands; NIR = near infrared band; RE = red edge band.

\subsubsection{Pre-processing}

Prior to photogrammetric processing, images were corrected for vignetting lens distortion and sensor variability. All 2014 imagery was then processed into orthomosaics by aerial survey and photogrammetric company, Aerometrex PL. All 2015 imagery was processed by Supervising Scientist staff using Pix4DMapper. Processing of UAS data typically involves several steps (Strecha et al. 2012). Firstly, the geometric alignment of photos using a feature matching algorithm and the image position and orientation based on the autopilots photolog are used to conduct a bundle block adjustment. Based on this reconstruction, a 3D point cloud is created which is interpolated forming a mesh which provides a digital surface model (DSM). The DSM is then used to project every image pixel and a georeferenced orthomosaic is calculated. RGB and NIR data were stacked to create 4 band images. Finally, the data were radiometrically calibrated to surface reflectance using the known reflectance of the panels and an empirical line method (Smith and Milton 1999).

\subsubsection{Analysis}

The analysis workflow applied to the imagery from each date is shown in Figure 4. The first step consisted of the calculation of a vegetation index. The normalised difference vegetation index (NDVI) was created for all dates with NIR data. For the one date with no NIR data a surrogate, the Triangular Greenness 
Index (Hunt et al. 2013), was used. Each index layer was then subjected to a $5 \times 5$ Gaussian low pass convolution filter to remove noise. The filtered index was then segmented based on a threshold to create a layer of objects (L1) representative of plant cover on the site. The value of the vegetation index threshold variable was set at 0 . Objects that contained pixels with values greater than the threshold were classified vegetation cover, while those less than the threshold were classified as background.

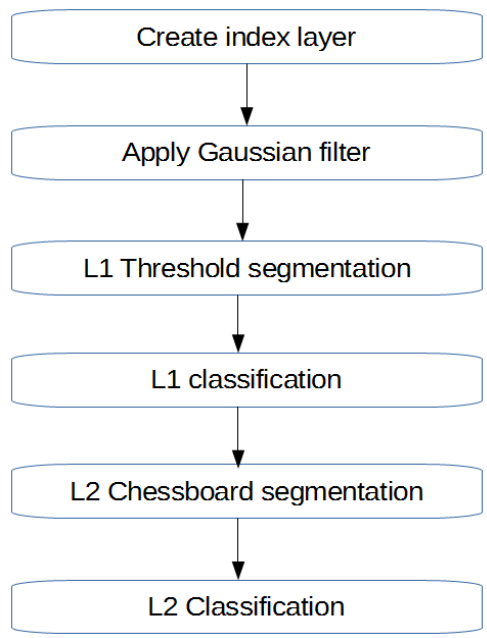

Figure 4. Image analysis workflow

A second layer (L2) of super-objects was created using a chessboard segmentation to create a grid of $10 \times 10 \mathrm{~m}$ objects (Figure 5). Proportional cover of L1 plant objects was then calculated per L2 object. Each L2 object was then assigned to a class based on the proportion of L1 plants sub objects: Less than $1 \%, 1-10 \%, 11-20 \%, 21-30 \%, 31-40 \%, 41-50 \%$, and greater than $50 \%$. Along with proportional cover, the number of plant objects and the average size of plant objects per L2 object were calculated. Change analysis was then conducted between dates on the L2 objects.

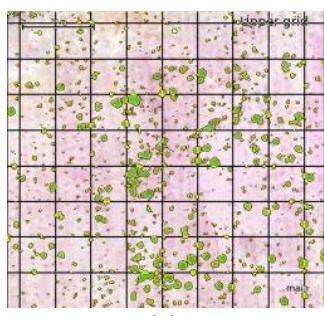

(a)

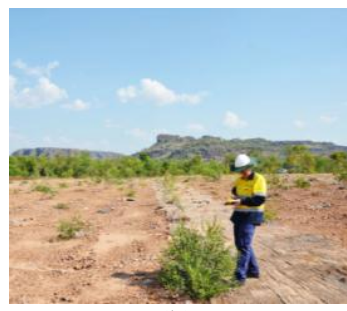

(b)
Figure 5. (a) A sample of the study area showing L1 objects (plants) and L2 objects (10 x $10 \mathrm{~m}$ grid). (b) Collecting field data.

\subsubsection{Validation}

Validation of accuracy was conducted using two methods. Field data were collected during the September 2014 capture, plant location (differential GNSS) and condition recorded for a transect across the site (Figure 5b). This data were compared to the L1 vegetation cover layer for that date. Further validation for other dates was conducted comparing the proportional cover for a random sample of $\mathrm{L} 2$ objects derived from this study to proportional cover estimated from manual aerial photointerpretation.

\section{RESULTS AND DISCUSSION}

All trees that were recorded in the field along the transect were detected in the L1 woody cover layer. There was also some commission error $(<5 \%)$ as a number of non tree objects were detected. These tended to be felt mats that were used as a mulch layer for the tubestock. The comparison between this objectbased proportional cover and the manually derived cover estimates also showed strong correlation $\left(r^{2}>0.8\right)$. Figure 6 shows the proportional cover maps for the mine site for each of the seven dates. The time series shows the change in cover over time highlighting an increase in relative cover within the eastern portion of the site. The maps also show a seasonal influence on the cover, with green growth during the wet season and some senescence in mature plants and mortality in juveniles during the drier months. A snapshot of 30 randomly selected L2 objects shows a trend of increasing relative cover from June 2014 onwards (Figure 7). The mean relative cover increased from less than 5\% in June 2014 to over 20\% in October 2015. Over the same time period however, there has been a decrease in the number of plant objects per L2 object (Figure 8). The mean number of L1 objects per L2 object was 44 in June 2014 decreasing to just over 16 in October 2015. This suggests that while there is plant mortality, the plants that are surviving are growing. It may also suggest that plant crowns are starting to overlap. The peak (April 2015) and trough (August 2015) in Figure 7 may be associated with the second planting (November 2014) and subsequent mortality through the dry season. Alternatively, the spike might be an artefact of the use of a different index for the April 2015 imagery. This will require further analysis. In addition, there was an observed natural recruitment of volunteer plants on site.
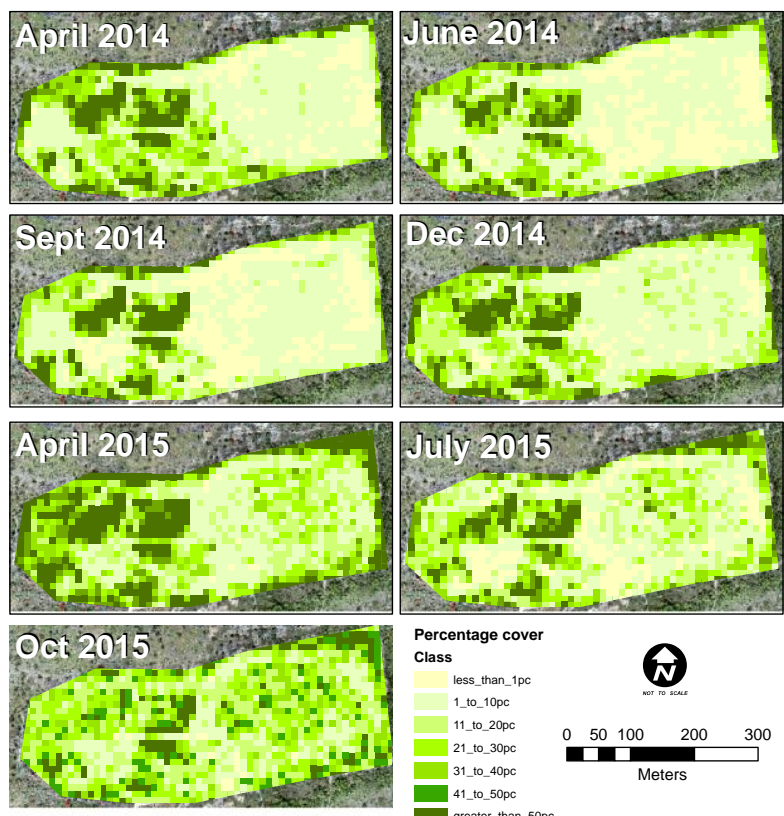

Figure 6. Proportional cover maps for the seven dates of image capture.

\section{CONCLUSION}

The study undertaken here has shown that multispectral UAS data of ultra-high spatial and temporal resolution can be used to provide information relevant to monitoring of mine site rehabilitation progress. Relevant bio-physical parameters, particularly the proportion of vegetation cover for the whole of site can be measured and assessed over time. Information such 
as this will be useful in determining whether a rehabilitation project is capable of achieving its desired outcomes. Further analysis will include a rigorous accuracy assessment of the time series analysis, the addition of more recent image captures, and whether it is possible to use the data to discriminate species. In addition, an assessment of the how much of the regrowth is from planted tubestock versus natural recruitment might also be useful.

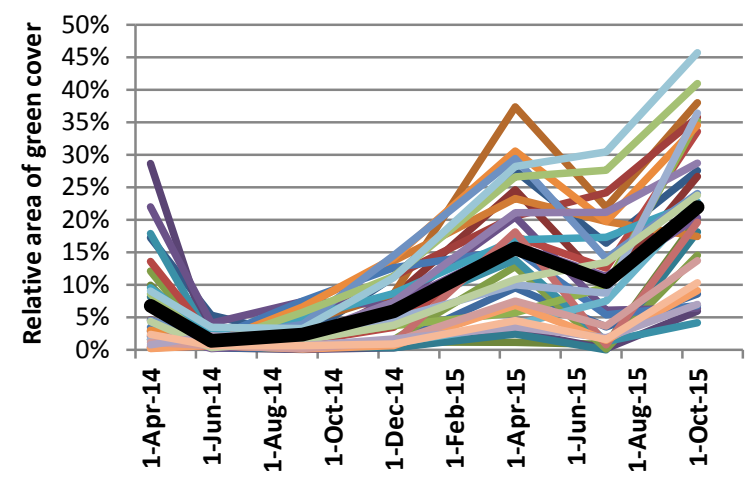

Figure 7. Change over time in the relative cover per the 30 randomly selected L2 objects. Thick black line is the mean relative cover for the objects selected.

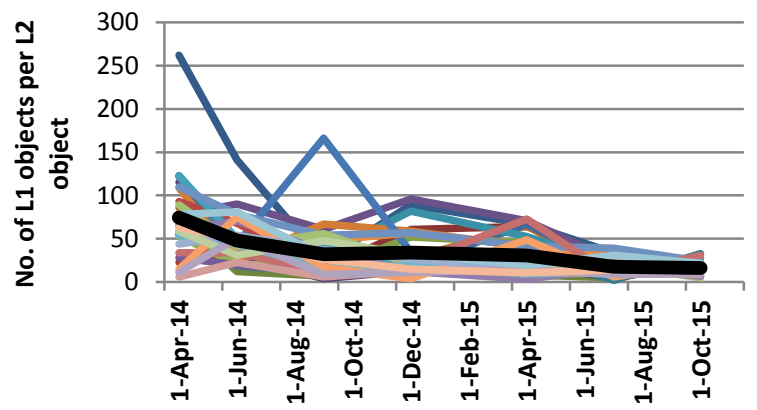

Figure 8. Change over time in the number of L1 objects per the 30 randomly selected L2 objects. Thick black line is the mean value for the objects selected.

\section{ACKNOWLEDGEMENTS}

Sandra Grant, Kirrilly Pfitzner and Andrew Esparon from the Supervising Scientist for field assistance.

\section{REFERENCES}

Fletcher, A.T., \& Erskine, P.D. 2013. Rehabilitation closure criteria assessment using high resolution photogrammetrically derived surface models. International Archives of Photogrammetry, Remote Sensing and Spatial Information Sciences, $X L-1 / W 2$, pp. 137-140.

Hunt, E.R., Doraiswamy, P.C., McMurtrey, J.E., Daughtry, C.S., Perry, E.M., \& Akhmedov, B. 2013. A visible band index for remote sensing leaf chlorophyll content at the canopy scale. International Journal of Applied Earth Observation and Geoinformation, 21, pp. 103-112.
Smith, G.M., \& Milton, E.J. 1999. The use of the empirical line method to calibrate remotely sensed data to reflectance. International Journal of Remote Sensing, 20, pp. 2653-2662.

Strecha, C., Bronstein, A.M., Bronstein, M.M., \& Fua, P. 2012. LDAHash: Improved matching with smaller descriptors. IEEE Transactions on Pattern Analysis and Machine Intelligence, 34, pp. 66-78. 